\title{
Pathophysiological Role of TRPM2 in Age-Related Cognitive Impairment in Mice
}

$\operatorname{AUTHOR}(S)$ :

Kakae, Masashi; Miyanohara, Jun; Morishima, Misa; Nagayasu, Kazuki; Mori, Yasuo; Shirakawa, Hisashi; Kaneko, Shuji

\section{CITATION:}

Kakae, Masashi ...[et al]. Pathophysiological Role of TRPM2 in Age-

Related Cognitive Impairment in Mice. Neuroscience 2019, 408: 204-213

\section{ISSUE DATE:}

2019-06-01

URL:

http://hdl.handle.net/2433/241618

\section{RIGHT:}

(c) 2019. This manuscript version is made available under the CC-BY-NC-ND 4.0 license

http://creativecommons.org/licenses/by-nc-nd/4.0/; The full-text file will be made open to the public on 1 June 2020 in accordance with publisher's 'Terms and Conditions for Self-Archiving'.; This is not the published version. Please cite only the published version.; この論文は出版社版でありません。引用の際には出版社版をご確認ご利用ください。 


\section{Pathophysiological role of TRPM2 in age-related cognitive impairment in} mice

Masashi Kakae, ${ }^{1 *}$ Jun Miyanohara, ${ }^{1 *}$ Misa Morsishima, ${ }^{1}$ Kazuki Nagayasu, ${ }^{1}$ Yasuo Mori, ${ }^{2}$ Hisashi Shirakawa ${ }^{1 \#}$ \& Shuji Kaneko ${ }^{1}$

${ }^{1}$ Department of Molecular Pharmacology, Graduate School of Pharmaceutical Sciences, Kyoto University, 46-29 Yoshida-Shimoadachi-cho, Sakyo-ku, Kyoto 606-8501, Japan

${ }^{2}$ Department of Synthetic Chemistry and Biological Chemistry, Graduate School of Engineering, Kyoto University, Katsura Campus, Nishikyo-ku, Kyoto 615-8510, Japan *M.K. and J.M. contributed equally to this study as co-first authors.

${ }^{\#}$ Corresponding author:

Hisashi Shirakawa, PhD, 46-29 Yoshidashimoadachicho, Sakyo, Kyoto 606-8501, Japan Tel.: +81-75-753-4549; Fax: +81-75-753-4548; E-mail: shirakaw@pharm.kyoto-u.ac.jp 


\section{Abstract}

Aging causes various functional changes, including cognitive impairment and inflammatory responses in the brain. Transient receptor potential melastatin 2 (TRPM2), a $\mathrm{Ca}^{2+}$-permeable channel expressed abundantly in immune cells, exacerbates inflammatory responses. Previously, we reported that TRPM2 on resident microglia plays a critical role in exacerbating inflammation, white matter injury, and cognitive impairment during chronic cerebral hypoperfusion; however, the physiological or pathophysiological role of TRPM2 during age-associated inflammatory responses remains unclear. Therefore, we examined the effects of TRPM2 deletion in young (2-3 months) and older (12-24 months) mice. Compared with young wild-type (WT) mice, middle-aged (12-16 months) WT mice showed working and cognitive memory dysfunction and aged (20-24 months) WT mice exhibited impaired spatial memory. However, these characteristics were not seen in TRPM2 knockout (TRPM2-KO) mice. Consistent with the finding of cognitive impairment, aged WT mice exhibited white matter injury and hippocampal damage and an increase in the number of Iba1-positive cells and amounts of pro-inflammatory cytokines in the brain; these characteristics were not seen in TRPM2-KO mice. These findings suggest that TRPM2 plays a critical role in exacerbating inflammatory responses and cognitive dysfunction during aging.

\section{Six key words:}

Aging; Cognitive impairment; White matter injury; TRPM2; Microglia; Cytokines. 


\section{Introduction}

Aging is defined broadly as the time-dependent functional decline in cardiac, renal, cognitive, and immune function (López-Otín et al., 2013; Young 1997). Cognitive impairment is one of the most significant factors in terms of its social and economic impact on not only elderly individuals but also on families and/or caregivers (Sharma et al., 2010). Often, this condition is accompanied by more serious aging-associated diseases such as Alzheimer’s disease and vascular dementia (Gorelick et al., 2011).

Several lines of evidence suggest that age-related vascular diseases are associated with altered inflammatory responses, particularly increased production of pro-inflammatory cytokines (Grammas and Ovase, 2001; Bruunsgaard et al., 2001). A previous report shows that chronic cerebral hypoperfusion-induced cognitive impairment in mice (Saggu et al., 2016) and humans (Kawamoto et al., 2006) is highly associated with inflammation. Moreover, anti-inflammatory drugs reduce cognitive impairment in a rodent vascular dementia model (Fu et al., 2014); they also reduce the incidence of Alzheimer’s disease (In'T Veld et al., 2001).

Recent studies suggest that inflammatory responses increase during normal aging, even in individuals without overt neurodegenerative disease (Salminen et al., 2012). Increased expression of pro-inflammatory genes is observed in aged tissues in rodents (Csiszar et al., 2003; Swindell et al., 2009) and humans (De Magalhães et al., 2009). Moreover, subacute treatment with ibuprofen rescues synaptic and cognitive deficits in aged mice (Rogers et al., 2017), suggesting that regulating inflammatory response is a potential method of arresting cognitive decline during normal aging.

Microglia are immune cells resident in the central nervous system (CNS) (Li and Barres, 2018). Studies show that dystrophic changes occur in the aged human brain 
(Ransohoff, 2016). Repopulation of microglia after elimination by PLX5622, a colony-stimulating factor 1 receptor inhibitor, restores microglial morphology (repopulated cells resemble young cells) and ameliorates cognitive dysfunction (Elmore et al., 2018). These reports imply that functional alterations to microglia play important roles in aging-associated pathologies.

Transient receptor potential melastatin 2 (TRPM2), a member of the TRPM superfamily, is a $\mathrm{Ca}^{2+}$-permeable channel with a unique C-terminal adenosine diphosphate ribose pyrophosphatase domain (Perraud et al., 2001). TRPM2 is functionally expressed in the brain, including by neurons (Kaneko et al., 2006) and microglia (Kraft et al., 2004; Miyake et al., 2014), and by peripheral inflammatory cells such as monocytes/macrophages (Yamamoto et al., 2008) and neutrophils (Hiroi et al., 2012). In addition, TRPM2 plays a role in production of pro-inflammatory cytokines/chemokines by monocytes/macrophages, and in exacerbation of inflammation-related disorders such as ulcerative colitis (Yamamoto et al., 2008) and neuropathic pain (Haraguchi et al., 2012). Moreover, TRPM2 plays an important role in $\beta$-amyloid-mediated neuronal toxicity and memory impairment in an Alzheimer's mouse model (Ostapchenko et al., 2015). Recently, we demonstrated white matter injury and cognitive dysfunction in a chronic cerebral hypoperfusion mouse model in which TRPM2-mediated activation of microglia plays a critical role in inducing cognitive impairment (Miyanohara et al., 2018). Although these reports imply that TRPM2 plays a role in chronic inflammation and cognitive dysfunction during aging, the physiological role of TRPM2 remains unclear.

Here, we used genetically engineered mice to investigate the possible involvement of TRPM2 in aging, and evaluated the effects of TRPM2 deficiency on age-dependent 
cognitive dysfunction. 


\section{Materials and Methods}

\section{Animals}

All experiments were conducted in accordance with the ethical guidelines of the Kyoto University animal experimentation committee and with the guidelines of the Japanese Pharmacological Society. Male C57BL/6J wild-type (WT) mice (RRID: IMSR_JAX:000664) and TRPM2-KO mice (2-24 months old) were maintained in-house. TRPM2-KO mice were generated as reported previously (Yamamoto et al., 2008) and backcrossed with C57BL/6J mice (Japan SLC) for ten generations to eliminate any background effects on the phenotype. All mice were housed at a constant ambient temperature of $22 \pm 2{ }^{\circ} \mathrm{C}$ under a $12 \mathrm{~h}$ light/dark cycle and were allowed water and chow ad libitum.

\section{Y-maze test}

The Y-maze test was conducted using mice aged 2-3 and 12-16 months of age. The Y-maze has three arms (40 cm long, $12 \mathrm{~cm}$ high, and $3 \mathrm{~cm}$ wide) arranged at equal angles. The arm into which the mice were placed initially was labeled A; the other two arms were labeled B and C. Experiments were performed over an 8 min period, and the total number and sequence of arm entries were recorded with a video camera. Alternation behavior was defined (and counted) as a mouse entering all three of the arms consecutively ( $\mathrm{ABC}, \mathrm{CAB}$, or $\mathrm{BCA}$, but not $\mathrm{BAB}$ ). The alternation count was divided by the maximum number of alternations (the total number of entries minus 2) and multiplied by 100 to create a percentage. The total number of arm entries was recorded as "spontaneous activity". Mice that made $<15$ arm entries were excluded. 


\section{Novel object recognition test (NORT)}

Cognitive assessment using the NORT was performed using young (2-3 months) and middle-aged (12-16 months) mice. Experiments were conducted under dim illumination (30 Lux). First, mice were habituated to a black box $(30 \times 30 \times 30 \mathrm{~cm})$ for 3 days (10 min per day) before training. During the training session, two different objects (a yellow triangular prism and a blue quadrangular pyramid) were placed in the box; the mice were allowed to interact freely with the objects for $10 \mathrm{~min}$. Six hours after the training session, the test session was performed whereupon the blue quadrangular object was replaced by a wooden ball, which served as a "novel object". Total exploratory time was defined as the time spent exploring both objects, and was considered an indicator of locomotor activity. Exploratory preference was defined as the ratio of the time spent exploring the blue quadrangular object during the training session or the wooden ball in the test session versus the total time spent exploring both objects. The result was considered to be an indicator of recognition memory.

\section{Novel location recognition test (NLRT)}

The test apparatus and the habituation/training procedure were identical to those described for the NORT. During the test session, the blue quadrangular object was moved to a different location. As mentioned above, total exploratory time was defined as the time spent exploring both objects and was considered an indicator of locomotor activity. Exploratory preference was defined as the ratio of the time spent exploring the blue quadrangular object versus the total time spent exploring both objects. This is considered to be an indicator of spatial memory. 


\section{Myelin staining}

Mice were injected intraperitoneally with pentobarbital $(50 \mathrm{mg} / \mathrm{kg})$ and then perfused transcardially with $0.1 \mathrm{M}$ PBS, followed by 4\% paraformaldehyde in $0.1 \mathrm{M}$ PBS. Brains were stored in the fixative for $3 \mathrm{~h}$ and then transferred to $15 \%$ sucrose in $0.1 \mathrm{M}$ phosphate buffer for $24 \mathrm{~h}$. Coronal sections (20 $\mu \mathrm{m}$ thick) were cut using a cryomicrotome To stain myelin, brain sections were soaked in $0.1 \%$ Triton X-100 for 20 min and then incubated for 20 min at room temperature with Fluoromyelin Green fluorescent myelin stain (1:300; Invitrogen). Fluorescence was visualized under an Olympus Fluoview microscope equipped with a laser scanning confocal imaging system. The mean intensity of myelin staining in the corpus callosum was measured in a $200 \times$ $200 \mu \mathrm{m}$ field sited at approximately bregma $+0.7 \mathrm{~mm}$.

\section{Immunofluorescence}

Coronal sections were prepared as described previously and incubated overnight at $4^{\circ} \mathrm{C}$ with primary antibodies specific for GSTpi (rabbit anti-GSTpi antibody, 1:200; MBL Life Science), Iba1 (rabbit anti-Iba1 antibody, 1:500; Wako Pure Chemical Industries), or CD3 (rat anti-CD3 antibody, 1:100; BD Biosciences). Next, sections were labeled with fluorescence-conjugated secondary antibodies (Alexa Fluor 594-labeled donkey anti-rabbit or Alexa Fluor 488-labeled donkey anti-rat IgG, 1:300; Invitrogen) at room temperature for $1.5 \mathrm{~h}$ in the dark. Images were captured under a confocal fluorescence microscope. GSTpi-, Iba1-, and CD3-positive cells in a 0.125 $\mathrm{mm}^{2}$ field of the corpus callosum at bregma $+0.7 \mathrm{~mm}$ and in the hippocampus at bregma - $2.8 \mathrm{~mm}$ were counted. 


\section{NeuN staining}

Coronal sections were incubated at $4^{\circ} \mathrm{C}$ overnight with a primary antibody specific for NeuN (mouse anti-NeuN antibody, 1:500; Chemicon) and then labeled for $1.5 \mathrm{~h}$ with biotinylated horse anti-mouse IgG (1:200, Vector Laboratories). Next, sections were incubated for $1 \mathrm{~h}$ in $\mathrm{ABC}$ Elite reagent (1:200, Vector Laboratories). Immunoreactivity was visualized using diaminobenzidine (Dojindo) as the chromogen. The number of NeuN-positive cells in the hippocampus at bregma $-2.8 \mathrm{~mm}$ was evaluated.

\section{Real-time PCR}

To isolate the corpus callosum and hippocampus, coronal slices at 2-mm interval were made in preparation of subsequent dissections. The dissected brain regions were immediately frozen in liquid nitrogen and stored at $-80{ }^{\circ} \mathrm{C}$ until use. Total RNA was isolated using ISOGEN reagent (Nippon Gene) in accordance with the manufacturer's suggested protocols, and cDNA was synthesized from $1 \mu \mathrm{g}$ of total RNA using ReverTra Ace® (Toyobo). Real-time quantitative PCR was performed using the StepOne real-time PCR system (Life Technologies, Carlsbad, CA, USA). The final reaction volume was $20 \mu \mathrm{l}$ (25 ng of cDNA plus THUNDERBIRD SYBR qPCR Mix, Toyobo). The PCR conditions were as follows: heating for $10 \mathrm{~min}$ at $95^{\circ} \mathrm{C}$, followed by 40 cycles at $95^{\circ} \mathrm{C}$ for $10 \mathrm{~s}$ and $60^{\circ} \mathrm{C}$ for $1 \mathrm{~min}$. The following oligonucleotide primers were used: IL1 $\beta$, 5'-TGA GCA CCT TCT TTT CCT TCA-3' and 5'-TTG TCT AAT GGG AAC GTC ACA C-3'; IL6, 5'-GTG GCT AAG GAC CAA GAC CA-3' and 5'-TAA CGC ACT AGG TTT GCC GA-3'; TNFa, 5'-TGC CTA TGT CTC AGC CTC TTC-3' and 5'-GAG GCC ATT TGG GAA CTT CT-3'; and 18S, 5'-GCA ATT ATT 
CCC CAT GAA CG-3' and 5'-GGC CTC ACT AAA CCA TCC AA-3'. The amount of 18S rRNA in samples was used to normalize the mRNA content (the mRNA level was expressed relative to that of the corresponding control).

\section{Experimental design and statistical analysis}

Statistical analysis was performed using Prism 7 software (GraphPad Software Inc.). One-way ANOVA followed by Tukey's post-hoc comparison test was used to compare data from multiple experimental groups. In all cases, differences of $\mathrm{p}<0.05$ were considered statistically significant. Data are expressed as the mean \pm SEM. Each data point represents a single sample (sections or extracts from specific brain regions such as the corpus callosum or hippocampus) from a single mouse. The number of animals used in each experiment is indicated in the figure legends. The assessor was blinded to treatment conditions. 


\section{Results}

Assessment of cognitive impairment with aging

First, we performed tests designed to compare behaviors of mice aged 2-3 months (young) with those of mice aged 12-16 months (middle-aged). Apart from an increase in body weight with age, there was no difference between the physical characteristics of WT and TRPM2-KO mice (data not shown). Additionally, our recent study demonstrated that TRPM2-KO mice did not differ significantly from WT mice in total distance, vertical activity, center time, or stereotypic counts in the open field test, indicating that TRPM2-KO mice possess normal locomotor activity (Andoh et al., 2019). Next, we conducted the Y-maze test to assess the role of TRPM2 in age-related cognitive impairment (Fig. 1A). Middle-aged mice made fewer arm entries (an index of locomotor activity) than young mice (data not shown). In addition, alternation behavior (an indicator for working memory) decreased in middle-aged WT mice; this decrease was markedly attenuated in middle-aged TRPM2-KO mice (Fig. 1A). To examine cognitive performance further, we performed a novel object recognition test to assess recognition memory (Fig. 1B). We found that the total time taken for middle-aged WT mice to explore two different objects was no different from that taken by TRPM2-KO mice (data not shown). Mice spent about $50 \%$ of the time exploring each object, with no significant difference being observed between training sessions (data not shown). However, when one object was replaced with a different one the time spent by middle-aged WT mice exploring the novel object was shorter than that spent by middle-aged TRPM2-KO mice (Fig. 1B). Next, we conducted a novel location recognition test to evaluate spatial memory function in middle-aged mice (Fig. 1C). Although exploratory preferences for two different objects during the training session 
were at chance levels (50\%) (data not shown), preference for exploring the relocated object during the test session was $>50 \%$, with no difference observed between the young and middle-aged groups (WT and TRPM2-KO) (Fig. 1C). These findings suggest that working and recognition memory are impaired, but spatial memory is preserved, in middle-aged mice, and that TRPM2 may play a role in development of memory dysfunction.

Next, we examined the spatial memory function of mice aged 20-24 months (Fig. 1D). These mice showed the same preference for both objects in the training session (data not shown); however, aged WT mice spent significantly less time exploring the newly located object than young WT mice. Again, this was reversed in aged TRPM2-KO mice (Fig. 1D). These results imply that aging induces spatial memory dysfunction, and that the underlying mechanism involves TRPM2.

\section{Evaluation of age-related white matter injury and hippocampal damage}

To investigate the association between TRPM2 and aging further, we examined histological changes in young (2-3 months) and aged (20-24 months) mice. We focused on brain regions involved in cognitive dysfunction. White matter is one of the most important parts of the brain in terms of working memory (Nishio et al., 2010), non-spatial recognition memory (Pitsikas and Tarantilis, 2017), and episodic memory (Lockhart et al., 2012). In cases of dementia (Douaud et al., 2013) and other psychological disorders (de Groot, 2000), white matter damage is highly associated with cognitive impairment. Therefore, to evaluate involvement of TRPM2 in age-related white matter injury, we stained myelin in the corpus callosum (Fig. 2A). Although, there were no apparent differences in white matter integrity between young WT and 
TRPM2-KO mice (Miyanohara et al., 2018), myelin density in aged WT mice was significantly lower than that in young WT mice. By contrast, no such decrease was observed in aged TRPM2-KO mice (Fig. 2B). To further assess white matter damage, we stained brain sections for GSTpi, a marker of oligodendrocytes (Fig. 2C). The number of GSTpi-positive cells in the corpus callosum of WT mice decreased with age (similar to myelin density); again, no such decrease was observed in TRPM2-KO mice (Fig. 2D). These results imply that TRPM2 plays a critical role in aggravating white matter injury.

Next, to further investigate the mechanism underlying cognitive dysfunction, we examined the hippocampus, which plays an important role in cognitive function, including spatial memory (Vago and Kesner, 2008; Shi et al., 2015). We stained brain sections for the neuron marker NeuN to assess neuronal survival in the hippocampal area (Fig. 3A). The number of NeuN-positive cells in the dentate gyrus (DG) did not change with age (Fig. 3B). However, the numbers in the CA1 and CA3 regions of aged WT mice were significantly lower than those in young WT mice; there was no difference between aged TRPM2-KO mice and young WT mice (Fig. 3B). These findings indicate that TRPM2 may play a role in neuronal loss in the hippocampus.

\section{Role of Iba1- and CD3-positive cells in the white matter and hippocampus}

Next, we examined expression of the microglia/macrophage marker Iba1 to identify the cells responsible for exacerbating damage to the white matter and hippocampus (Fig. 4). We also examined expression of the T lymphocyte marker CD3 (Fig. 5) in the corpus callosum and hippocampus. The number of Iba1-positive cells in the corpus callosum of aged WT mice was higher than that in young WT mice, while that in aged 
TRPM2-KO mice was similar to that in young mice (Fig. 4A, B). Similarly, the number of Iba1-positive cells in the hippocampus of WT mice increased significantly with age; in addition, the number in the DG, CA1, and CA3 of TRPM2-KO mice tended to be lower than that in aged WT mice (Fig. 4C, D).

By contrast, the number of CD3-positive cells in the corpus callosum (Fig. 5A, B) and hippocampus (Fig. 5C, D) increased with age, although there was no difference between WT and TRPM2-KO mice. Taken together, these results suggest that damage to the white matter and hippocampus may be caused by inflammatory responses resulting from TRPM2-mediated activation of microglia.

Age-related changes in inflammatory responses in the corpus callosum and hippocampus

Next, we attempted to examine pathological changes in the white matter and hippocampal areas. To do this, we performed quantitative RT-PCR to detect mRNA encoding inflammatory cytokines such as IL1 $\beta$, IL6, and TNF $\alpha$ in the corpus callosum and hippocampus. Expression of IL1 $\beta$ (Fig. 6A), IL6 (Fig. 6B), and TNF $\alpha$ (Fig. 6C) in the corpus callosum of aged WT mice was significantly or tented to be higher than that in young WT mice; expression tended to be lower in aged TRPM2-KO mice. In addition, we observed a significant increase in expression of TNF $\alpha$ in the hippocampus of aged WT mice; expression tended to be lower in aged TRPM2-KO mice (Fig. 6F). Expression of IL1 $\beta$ (Fig. 6D) and IL6 (Fig. 6E) in aged TRPM2-KO mice was similar to that in aged WT. These results imply that inflammatory reactions play a role in white matter- and hippocampus-related cognitive impairment, which is mediated by activation of TRPM2. 


\section{Discussion}

The present study demonstrates that aged mice show impairment of working, recognition, and spatial memory. Moreover, to the best of our knowledge, we provide the first evidence that knocking out the TRPM2 gene attenuates cognitive dysfunction and prevents age-related damage to the white matter and hippocampus. In addition, we found that age-related inflammatory responses tended to be suppressed in TRPM2-KO mice. These results suggest that activation of TRPM2 has a deleterious effect on the pathophysiology of the aging brain.

Memory function is one of the most vulnerable age-related characteristics (Larrabee and Crook, 1994). Previous reports show that aging results in damage to the white matter and hippocampus (Stahon et al., 2016; Bastian et al., 2018; Bishop and Yankner, 2010). However, it is not clear whether one is damaged to a greater or lesser extent than the other. Other groups report that, in mice aged 18 months, the reduction in myelin is greater than that of neurites (Woodward et al., 2017). In addition, water maze tests reveal that 11-month-old mice show working memory dysfunction, whereas reference memory (hippocampus-related) is preserved (Das and Magnusson, 2011); this suggests that white matter-associated memory is particularly vulnerable. Rogers et al. demonstrate that reduced long-term potentiation (LTP) and spatial memory disturbance in the hidden platform water maze test occur at 24 months of age and later, but not at 18 months of age (Rogers et al., 2017). Similar to these observations, we detected spatial memory impairment in mice aged 24 months. Moreover, chronic cerebral hypoperfusion model mice show hippocampal atrophy (accompanied by reference memory dysfunction) much later than impairment of white matter-related working memory (Nishio et al., 2010). Taken together, the data suggest that it is conceivable that 
age-related cognitive dysfunction is involved in damage to the white matter and hippocampus, and that the white matter may be injured before the hippocampus.

Here, we show an age-related increase in the number of Iba1- and CD3-positive cells and in the amounts of pro-inflammatory cytokines. One of the key characteristics of brain aging is inflammation (Ojo et al., 2015). As inflammation develops, glial cells (particularly microglia) in the aged brain show marked phenotypic and functional changes not evident in the young brain (Raj et al., 2017; Martin et al., 2017; Friedman et al., 2018); these include increased cell numbers, dystrophic morphology, impaired phagocytosis, reduced motility, and expression of molecules indicative of an inflammatory phenotype (Martin et al., 2017). Here, we used only immunohistochemical approaches to assess expression of Iba1; therefore, future work will examine detailed changes in microglial function.

A previous report demonstrates that CD11c-positive dendritic cells and CD3-positive $\mathrm{T}$ cells infiltrate the white matter region of aged mice (Stichel and Luebbert, 2007), indicating a potential role for leukocytes in age-related inflammatory responses in the brain. Another report revealed an increase in the numbers of ED1-positive macrophages in the brain of 18-month-old rats, and in the brain of 2-month-old rats after injection of TNF $\alpha$ into the striatum (Campbell et al., 2007). In addition, TNF $\alpha$ levels in the hippocampus of 12-month-old mice are higher than those in 2-month-old mice (Tichauer et al., 2014). Moreover, permeability of the blood-brain barrier in humans increases with age (Farrall and Wardlaw, 2009). Taken together, these reports suggest that age-related brain inflammation may be driven by migration of peripheral immune cells to the brain. The data presented in the present study suggest that knocking out the TRPM2 gene does not affect infiltration of CD3-immunopositive 
cells into the corpus callosum and hippocampus, implying that TRPM2 expressed by peripheral immune cells does not play an important role in inflammation of the aged brain.

Previous studies reveal that cytokine levels in mice increase with age (Moranis et al., 2012); this is true even in healthy elderly adults (Singh and Newman, 2011). Moreover, Deczkowska et al. demonstrated marked upregulation of mRNA encoding IL1 $\beta$ and TNF $\alpha$, and of that encoding CCLs, in microglia of aged mice when compared with young mice (Deczkowska et al., 2017). Here, we detected upregulation of TNF $\alpha$ mRNA in the corpus callosum and hippocampus of aged mice; however, the expression tended to be lower in aged TRPM2-KO mice. Taken together, the data suggest that TRPM2-mediated activation of immune cells might induce inflammatory signals, which damages myelin and neurons in the aged brain, eventually resulting in cognitive dysfunction. Future studies are needed to examine the role of other pro-inflammatory cytokines in the aged brain.

We also showed that deleterious age-related events were significantly (or tended to be) suppressed in aged TRPM2-KO mice. One may ask which mechanisms contribute to (and more importantly why knocking out TRPM2 suppresses) cognitive impairment in aged mice. In a previous report, we demonstrated cytokine-induced damage to white matter and cognitive dysfunction in a chronic cerebral hypoperfusion mouse model; release of these cytokines is triggered mainly by TRPM2-mediated activation of microglia (Miyanohara et al., 2018). A previous report shows that cortical cerebral blood flow (CBF) in humans decreases with age (Aanerud et al., 2012), whereas another demonstrates attenuation of CBF responses and an increase in production of reactive oxygen species (ROS) in 18- and 24-month-old mice compared with 3-month-old mice 
(Park et al., 2014). Taken together, these reports suggest that, because TRPM2 is activated by ROS, these species could induce age-related cognitive dysfunction and inflammatory responses in the aged brain via TRPM2. Because white matter is vulnerable to ROS, and because white matter injury tends to occur before prior hippocampal injury, ROS could play a critical role in age-related pathology; however, future studies are needed to clarify the moieties that activate TRPM2 (and in which cell types) in the aged brain. In addition, previous reports demonstrate increased ROS production and neuronal death in aging rats (Ureshino et al., 2018). Moreover, others show that TRPM2 expressed by neurons plays a role in $\mathrm{H}_{2} \mathrm{O}_{2}$-induced neuronal death (Li and Jiang, 2017). Here, we found that the number of NeuN-positive cells in the hippocampus fell in aged WT mice but not in aged TRPM2-KO mice. Taken together, these results imply that neuronal death is induced by ROS, and that TRPM2 may be involved in age-related neuronal damage and subsequent cognitive dysfunction.

TRPM2 is expressed abundantly in microglia (Kraft et al., 2004; Miyake et al., 2014) and peripheral inflammatory cells such as monocytes/macrophages (Yamamoto et al., 2008) and neutrophils (Hiroi et al., 2012). Previous studies reveal that TRPM2 plays a role in production of pro-inflammatory cytokines/chemokines by monocytes/macrophages, and in exacerbation of inflammation-related disorders such as ulcerative colitis (Yamamoto et al., 2008) and neuropathic pain (Haraguchi et al., 2012); these data imply that TRPM2 plays a role in the chronic inflammation that underlies senescence, which is characterized by inflammatory processes similar to those observed in other CNS diseases. Furthermore, our recent study clarifies that TRPM2-mediated microglial activation exacerbates cognitive impairment caused by chronic cerebral hypoperfusion (Miyanohara et al., 2018). Here, we found that the increase in the 
number of Iba1-positive cells tended to be reversed in aged TRPM2-KO mice, suggesting that suppression of immune responses, particularly microglial activation, targeted by TRPM2 may abrogate neuroinflammation-related cognitive dysfunction during aging.

In conclusion, we show that TRPM2 plays a critical role, at least in part, in the development of inflammation during brain aging, and that this may be mediated by activation of immune cells such as microglia. Thus, TRPM2 could be a potential therapeutic target to slow aging or prevent age-related cognitive disorders. 


\section{Acknowledgments}

This study was supported by MEXT/JSPS KAKENHI Grant Numbers 17K19486 (to H.S.), 24390016 (to S.K.). This work was also supported by the Novartis Foundation, the Takeda Science Foundation, and the Kyoto University Research Development Program (Ishizue). 


\section{References}

Aanerud J, Borghammer P, Chakravarty MM, Vang K, Rodell AB, Jonsdottir KY, Møller A, Ashkanian M, Vafaee MS, Iversen P, Johannsen P, Gjedde A (2012) Brain energy metabolism and blood flow differences in healthy aging. Journal of Cerebral Blood Flow \& Metabolism 32: 1177-1187.

Andoh C, Nishitani N, Hashimoto E, Nagai Y, Takao K, Miyakawa T, Nakagawa T, Mori Y, Nagayasu K, Shirakawa H, Kaneko S (2019) TRPM2 confers susceptibility to social stress but is essential for behavioral flexibility. Brain research 1704: 68-77.

Bastian C, Zaleski J, Stahon K, Parr B, McCray A, Day J, Brunet S, Baltan S (2018) NOS3 Inhibition Confers Post-Ischemic Protection to Young and Aging White Matter Integrity by Conserving Mitochondrial Dynamics and Miro-2 Levels. Journal of Neuroscience 38: 6247-6266.

Bishop NA, Lu T, Yankner BA (2010) Neural mechanisms of ageing and cognitive decline. Nature 464: 529-535.

Bruunsgaard H, Pedersen M, Pedersen BK (2001) Aging and proinflammatory cytokines. Current opinion in hematology 8: 131-136.

Campbell SJ, Carare-Nnadi RO, Losey PH, Anthony DC (2007) Loss of the atypical inflammatory response in juvenile and aged rats. Neuropathology and applied neurobiology 33: 108-120.

Csiszar A, Ungvari Z, Koller A, Edwards JG, Kaley G (2003) Aging-induced proinflammatory shift in cytokine expression profile in coronary arteries. The FASEB journal 17: 1183-1185.

Das SR, Magnusson KR (2011) Changes in expression of splice cassettes of NMDA receptor GluN1 subunits within the frontal lobe and memory in mice during aging. Behavioural brain research 222: 122-133. 
de Groot JC, de Leeuw FE, Oudkerk M, Hofman A, Jolles J, Breteler MM (2000)

Cerebral white matter lesions and depressive symptoms in elderly adults. Archives of general psychiatry 57: 1071-1076.

De Magalhães JP, Curado J, Church GM (2009) Meta-analysis of age-related gene expression profiles identifies common signatures of aging. Bioinformatics 25: 875-881.

Deczkowska A, Matcovitch-Natan O, Tsitsou-Kampeli A, Ben-Hamo S, Dvir-Szternfeld R, Spinrad A, Singer O, David E, Winter DR, Smith LK, Kertser A, Baruch K, Rosenzweig N, Terem A, Prinz M, Villeda S, Citri A, Amit I, Schwartz M (2017) Mef2C restrains microglial inflammatory response and is lost in brain ageing in an IFN-I-dependent manner. Nature Communications 8: 717.

Douaud G, Menke RA, Gass A, Monsch AU, Rao A, Whitcher B, Zamboni G, Matthews PM, Sollberger M, Smith S (2013) Brain microstructure reveals early abnormalities more than two years prior to clinical progression from mild cognitive impairment to Alzheimer's disease. Journal of Neuroscience 33: 2147-2155.

Elmore MR, Hohsfield LA, Kramár EA, Soreq L, Lee RJ, Pham ST, Najafi AR, Spangenberg EE, Wood MA, West BL, Green KN (2018) Replacement of microglia in the aged brain reverses cognitive, synaptic, and neuronal deficits in mice. Aging cell e12832.

Farrall AJ, Wardlaw JM (2009) Blood-brain barrier: ageing and microvascular diseasesystematic review and meta-analysis. Neurobiology of aging 30: 337-352.

Friedman BA, Srinivasan K, Ayalon G, Meilandt WJ, Lin H, Huntley MA, Cao Y, Lee S-H, Haddick PCG, Ngu H, Modrusan Z, Larson JL, Kaminker JS, van der Brug MP, Hansen DV (2018) Diverse brain myeloid expression profiles reveal distinct microglial activation states and aspects of Alzheimer's disease not evident in mouse models. Cell reports 22: 832-847.

Fu X, Zhang J, Guo L, Xu Y, Sun L, Wang S, Feng Y, Gou L, Zhang L, Liu Y (2014) Protective role of luteolin against cognitive dysfunction induced by chronic 
cerebral hypoperfusion in rats. Pharmacology Biochemistry and Behavior 126: 122-130.

Gorelick PB, Scuteri A, Black SE, DeCarli C, Greenberg SM, Iadecola C, Launer LJ, Laurent S, Lopez OL, Nyenhuis D, Petersen RC, Schneider JA, Tzourio C, Arnett DK, Bennett DA, Chui HC, Higashida RT, Lindquist R, Nilsson PM, Roman GC, Sellke FW, Seshadri S (2011) Vascular contributions to cognitive impairment and dementia: a statement for healthcare professionals from the American Heart Association/American Stroke Association. Stroke 42: 2672-2713.

Grammas P, Ovase R (2001) Inflammatory factors are elevated in brain microvessels in Alzheimer's disease. Neurobiology of aging 22: 837-842.

Haraguchi K, Kawamoto A, Isami K, Maeda S, Kusano A, Asakura K, Shirakawa H, Mori Y, Nakagawa T, Kaneko S (2012) TRPM2 contributes to inflammatory and neuropathic pain through the aggravation of pronociceptive inflammatory responses in mice. Journal of Neuroscience 32: 3931-3941.

Hiroi T, Wajima T, Negoro T, Ishii M, Nakano Y, Kiuchi Y, Y Mori, Shimizu S (2012) Neutrophil TRPM2 channels are implicated in the exacerbation of myocardial ischaemia/reperfusion injury. Cardiovascular research 97: 271-281.

In'T Veld BA, Ruitenberg A, Hofman A, Launer LJ, van Duijn CM, Stijnen T, Breteler MM, Stricker BH (2001) Nonsteroidal antiinflammatory drugs and the risk of Alzheimer's disease. New England Journal of Medicine 345: 1515-1521.

Kaneko S, Kawakami S, Hara Y, Wakamori M, Itoh E, Minami T, Takada Y, Kume T, Katsuki H, Mori Y, Akaike, A. (2006). A critical role of TRPM2 in neuronal cell death by hydrogen peroxide. Journal of pharmacological sciences 101: 66-76.

Kawamoto Y, Akiguchi I, Tomimoto H, Shirakashi Y, Honjo Y, Budka H (2006) Upregulated expression of 14-3-3 proteins in astrocytes from human cerebrovascular ischemic lesions. Stroke 37: 830-835.

Kraft R, Grimm C, Grosse K, Hoffmann A, Sauerbruch S, Kettenmann H, Schultz G, Harteneck C (2004) Hydrogen peroxide and ADP-ribose induce TRPM2-mediated 
calcium influx and cation currents in microglia. American Journal of Physiology-Cell Physiology 286: C129-C137.

Larrabee GJ, Crook TH (1994). Estimated prevalence of age-associated memory impairment derived from standardized tests of memory function. International psychogeriatrics 6: 95-104.

Li Q, Barres BA (2018) Microglia and macrophages in brain homeostasis and disease. Nature Reviews Immunology 18: 225.

Li X, Yang W, Jiang LH (2017). Alteration in intracellular Zn2+ homeostasis as a result of TRPM2 channel activation contributes to ROS-induced hippocampal neuronal death. Frontiers in molecular neuroscience 10: 414.

Lockhart SN, Mayda AB, Roach AE, Fletcher E, Carmichael O, Maillard P, Schwarz CG, Yonelinas AP, Ranganath C, DeCarli C (2012) Episodic memory function is associated with multiple measures of white matter integrity in cognitive aging. Frontiers in Human Neuroscience 6: 56.

López-Otín C, Blasco MA, Partridge L, Serrano M, Kroemer G (2013) The hallmarks of aging. Cell 153: 1194-1217.

Martin E, Boucher C, Fontaine B, Delarasse C (2017) Distinct inflammatory phenotypes of microglia and monocyte-derived macrophages in Alzheimer's disease models: effects of aging and amyloid pathology. Aging cell 16: 27-38.

Miyake T, Shirakawa H, Kusano A, Sakimoto S, Konno M, Nakagawa T, Yasuo Mori, Kaneko S (2014) TRPM2 contributes to LPS/IFN $\gamma$-induced production of nitric oxide via the p38/JNK pathway in microglia. Biochemical and biophysical research communications 444: 212-217.

Miyanohara J, Kakae M, Nagayasu K, Nakagawa T, Mori Y, Arai K, Shirakawa H, Kaneko S (2018) TRPM2 channel aggravates CNS inflammation and cognitive impairment via activation of microglia in chronic cerebral hypoperfusion. Journal of Neuroscience 38: 3520-3533. 
Moranis A, Delpech JC, De Smedt-Peyrusse V, Aubert A, Guesnet P, Lavialle M, Joffre C, Layé, S (2012) Long term adequate n-3 polyunsaturated fatty acid diet protects from depressive-like behavior but not from working memory disruption and brain cytokine expression in aged mice. Brain, behavior, and immunity 26: 721-731.

Nishio K, Ihara M, Yamasaki N, Kalaria RN, Maki T, Fujita Y, Ito H, Oishi N, Fukuyama H, Miyakawa T, Takahashi R, Tomimoto H (2010) A mouse model characterizing features of vascular dementia with hippocampal atrophy. Stroke 41: 1278-1284.

Ojo JO, Rezaie P, Gabbott PL, Stewart MG (2015) Impact of age-related neuroglial cell responses on hippocampal deterioration. Frontiers in aging neuroscience 7: 57.

Ostapchenko VG, Chen M, Guzman MS, Xie YF, Lavine N, Fan J, Beraldo FH, Martyn AC, Belrose JC, Mori Y, MacDonald JF, Prado VF, Prado MA, Jackson MF. (2015) The transient receptor potential melastatin 2 (TRPM2) channel contributes to $\beta$-amyloid oligomer-related neurotoxicity and memory impairment. Journal of Neuroscience 35: 15157-15169.

Park L, Koizumi K, El Jamal S, Zhou P, Previti ML, Van Nostrand WE, Carlson G, Iadecola C (2014) Age-dependent neurovascular dysfunction and damage in a mouse model of cerebral amyloid angiopathy. Stroke 45: 1815-1821.

Perraud AL, Fleig A, Dunn CA, Bagley LA, Launay P, Schmitz C, Stokes AJ, Zhu Q, Bessman MJ, Penner R, Kinet JP, Scharenberg AM (2001) ADP-ribose gating of the calcium-permeable LTRPC2 channel revealed by Nudix motif homology. Nature 411: 595.

Pitsikas N, Tarantilis PA (2017) Crocins, the active constituents of Crocus sativus L., counteracted apomorphine-induced performance deficits in the novel object recognition task, but not novel object location task, in rats. Neuroscience letters 644: 37-42.

Raj D, Yin Z, Breur M, Doorduin J, Holtman IR, Olah M, Mantingh-Otter IJ, Van Dam D, De Deyn PP, den Dunnen W, Eggen BJL, Amor S, Boddeke E (2017) Increased 
white matter inflammation in aging-and Alzheimer's disease Brain. Frontiers in molecular neuroscience 10: 206.

Ransohoff RM (2016) How neuroinflammation contributes to neurodegeneration. Science 353: 777-783.

Rogers JT, Liu CC, Zhao N, Wang J, Putzke T, Yang L, Shinohara M, Fryer JD, Kanekiyo T, Bu G (2017) Subacute ibuprofen treatment rescues the synaptic and cognitive deficits in advanced-aged mice. Neurobiology of aging 53: 112-121.

Saggu R, Schumacher T, Gerich F, Rakers C, Tai K, Delekate A, Petzold GC (2016) Astroglial NF-kB contributes to white matter damage and cognitive impairment in a mouse model of vascular dementia. Acta neuropathologica communications 4: 76.

Salminen A, Kaarniranta K, Kauppinen A (2012) Inflammaging: disturbed interplay between autophagy and inflammasomes. Aging (Albany NY) 4: 166.

Sharma S, Rakoczy S, Brown-Borg H (2010) Assessment of spatial memory in mice. Life sciences 87: 521-536.

Shi Q, Colodner KJ, Matousek SB, Merry K, Hong S, Kenison JE, Frost JL, Le KX, Li S, Dodart JC, Caldarone BJ, Stevens B, Lemere CA (2015) Complement C3-deficient mice fail to display age-related hippocampal decline. Journal of Neuroscience 35: 13029-13042.

Singh T, Newman AB (2011) Inflammatory markers in population studies of aging. Ageing research reviews 10: 319-329.

Stahon KE, Bastian C, Griffith S, Kidd GJ, Brunet S, Baltan S (2016) Age-related changes in axonal and mitochondrial ultrastructure and function in white matter. Journal of Neuroscience 36: 9990-10001.

Stichel CC, Luebbert H (2007) Inflammatory processes in the aging mouse brain: participation of dendritic cells and T-cells. Neurobiology of aging 28: 1507-1521.

Swindell WR (2009) Genes and gene expression modules associated with caloric restriction and aging in the laboratory mouse. BMC genomics 10: 585. 
Tichauer JE, Flores B, Soler B, Eugenín-von Bernhardi L, Ramírez G, von Bernhardi R (2014) Age-dependent changes on TGF $\beta 1$ Smad3 pathway modify the pattern of microglial cell activation. Brain, behavior, and immunity 37: 187-196.

Ureshino RP, Costa AJ, Erustes AG, Pereira GJDS, Sinigaglia-Coimbra R, Smaili SS (2018) Effects of Aging in the Striatum and Substantia Nigra of a Parkinson's Disease Animal Model. Toxicologic pathology 46: 348-358.

Vago DR, Kesner RP (2008) Disruption of the direct perforant path input to the CA1 subregion of the dorsal hippocampus interferes with spatial working memory and novelty detection. Behavioural brain research 189: 273-283.

Woodward NC, Pakbin P, Saffari A, Shirmohammadi F, Haghani A, Sioutas C, Cacciottolo M, Morgan TE, Finch CE (2017) Traffic-related air pollution impact on mouse brain accelerates myelin and neuritic aging changes with specificity for CA1 neurons. Neurobiology of aging 53: 48-58.

Yamamoto S, Shimizu S, Kiyonaka S, Takahashi N, Wajima T, Hara Y, Negoro T, Hiroi T, Kiuchi Y, Okada T, Kaneko S, Lange I, Fleig A, Penner R, Nishi M, Takeshima H, Mori Y. (2008). TRPM2-mediated $\mathrm{Ca}^{2+}$ influx induces chemokine production in monocytes that aggravates inflammatory neutrophil infiltration. Nature medicine 14: 738.

Young A (1997) Ageing and physiological functions. Philosophical Transactions of the Royal Society of London B: Biological Sciences 352: 1837-1843. 
Figure legends

Figure 1. Age-associated cognitive decline and its reversal by knockout of the TRPM2 gene.

A, Alternation behavior by young (2-3 months) and middle-aged (12-16 months) mice in the Y-maze test. B, Exploratory preference of young (2-3 months) and middle-aged (12-16 months) mice for a novel object in NORT. C, D, Exploratory preference of young (2-3 months) and middle-aged (12-16 months) mice (C) and of young (2-3 months) and aged (20-24 months) mice (D) for a newly located object in NLRT. Values are expressed as the mean \pm SEM. A, $n=15-19 ; \mathbf{B}, \mathrm{n}=7-10 \mathbf{C}, \mathrm{n}=4-6 ; \mathbf{D}, \mathrm{n}=9-16$. ${ }^{* *} \mathrm{p}<0.01$ and ${ }^{* * *} \mathrm{p}<0.001$, vs. young mice (closed columns); ${ }^{\#} \mathrm{p}<0.05$ and ${ }^{\# \#} \mathrm{p}<$ 0.01 .

Figure 2. Age-associated white matter injury and its reversal by knockout of the TRPM2 gene.

Representative images (A) and summarized data (B) showing myelin staining to assess myelin density in the corpus callosum of aged (20-24 months) mice. Representative immunostained images (C) and summarized data (D) showing the number of GSTpi-positive cells in the corpus callosum of aged (20-24 months) mice. Scale bars: $100 \mu \mathrm{m}$. Values are expressed as the mean \pm SEM. B, $\mathrm{n}=3-6 ; \mathbf{D}, \mathrm{n}=6 .{ }^{*} \mathrm{p}<0.05$ and **p $<0.01$ vs. WT mice aged $2-3$ months.

Figure 3. Age-associated hippocampal neuronal death and its reversal by knockout of the TRPM2 gene. 
Representative images (A) and summarized data (B) showing NeuN immunostaining to examine hippocampal damage in the dentate gyrus (left), CA1 (center), and CA3 (right) areas of the hippocampus of mice aged 20-24 months. Scale bars: $100 \mu \mathrm{m}$. Values are expressed as the mean \pm SEM. A, $\mathrm{n}=6 ; \mathbf{B}, \mathrm{n}=4 .{ }^{*} \mathrm{p}<0.05$ and ${ }^{* *} \mathrm{p}<0.01$ vs. young WT mice.

Figure 4. Age-associated increase in the number of microglia/macrophages in the corpus callosum and hippocampus, and its amelioration by knockout of the TRPM2 gene.

Representative images (A) and summarized data (B) showing immunostaining for Iba1-immunopositive cells in the corpus callosum of aged (20-24 months) mice. Representative images (C) and summarized data (D) showing the numbers of Iba1-immunopositive cells in the dentate gyrus (left), CA1 (center), and CA3 regions (right) of the hippocampus of aged (20-24 months) mice. Scale bars: $100 \mu \mathrm{m}$. Values are expressed as the mean \pm SEM. $\mathrm{n}=4 .{ }^{*} \mathrm{p}<0.05$ and ${ }^{* *} \mathrm{p}<0.01$ vs. young WT mice.

Figure 5. Age-associated infiltration of the corpus callosum and hippocampus of WT and TRPM2-KO mice by CD3-positive cells.

Representative images (A) and summarized data (B) showing immunostaining of CD3-immunopositive cells in the corpus callosum of aged (20-24 months) mice. Representative images (C) and summarized data (D) showing the number of CD3-immunopositive cells in the dentate gyrus (left), CA1 (center), and CA3 regions (right) of the hippocampus of aged (20-24 months) mice. Scale bars: $100 \mu \mathrm{m}$. Values are expressed as the mean \pm SEM. $\mathrm{n}=3-4 .{ }^{*} \mathrm{p}<0.05$ and ${ }^{* *} \mathrm{p}<0.01$ vs. young WT 
mice.

Figure 6. Upregulation of pro-inflammatory cytokines and chemokines in the white matter and hippocampal regions of aged WT mice.

Expression of IL1 $\beta(\mathbf{A}, \mathbf{D})$, IL6 (B, E), and TNF $\alpha(\mathbf{C}, \mathbf{F})$ mRNA in the corpus callosum (A-C) and hippocampus (D-F) of aged (20-24 months) mice. Values are expressed as the mean \pm SEM. $\mathrm{n}=6-9 .{ }^{*} \mathrm{p}<0.05$ vs. young (closed columns) WT mice. 
Fig. 1
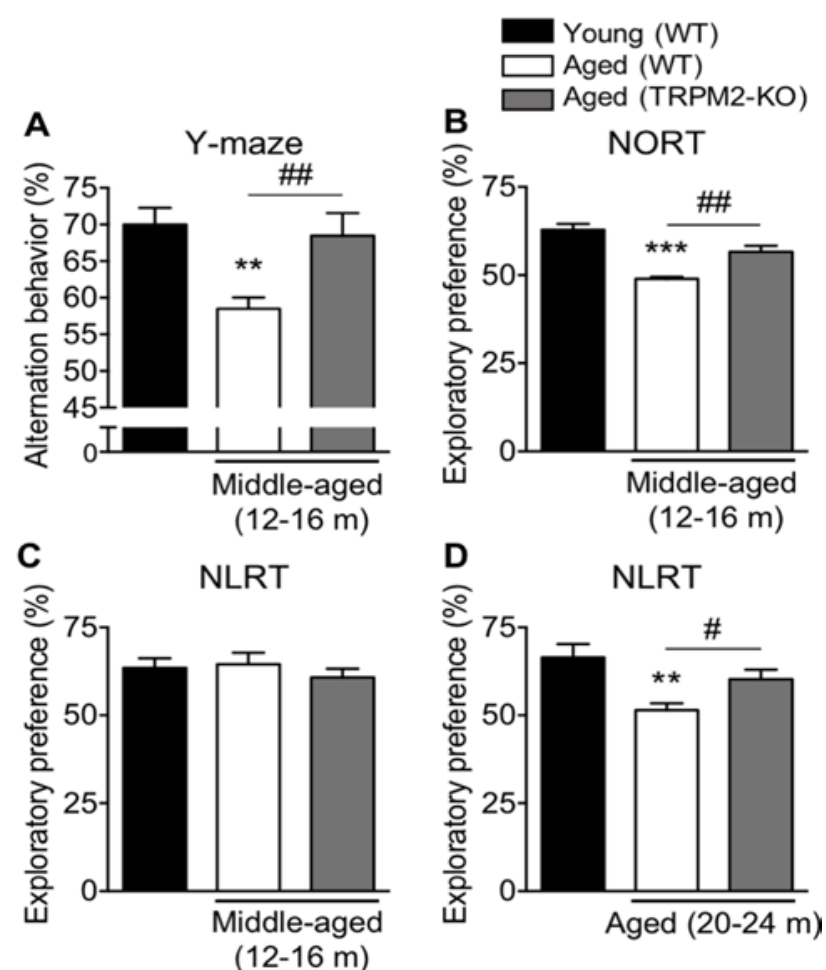

Fig. 2
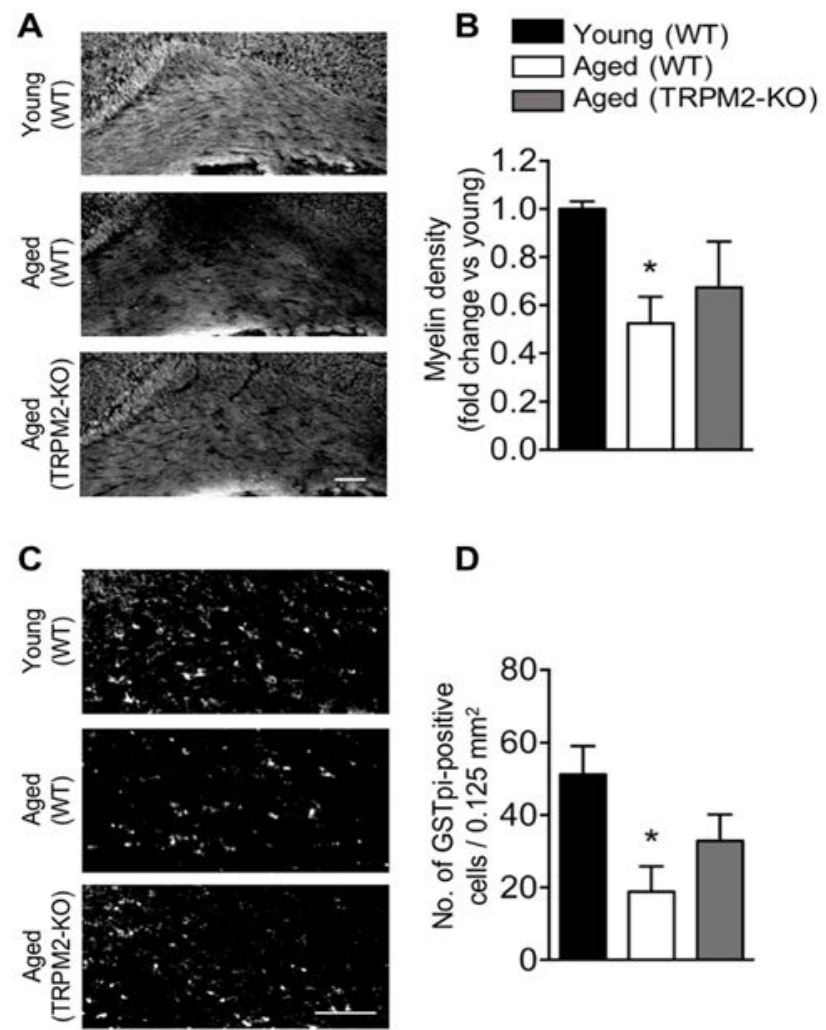

D

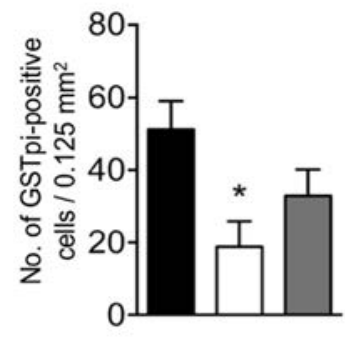


Fig. 3

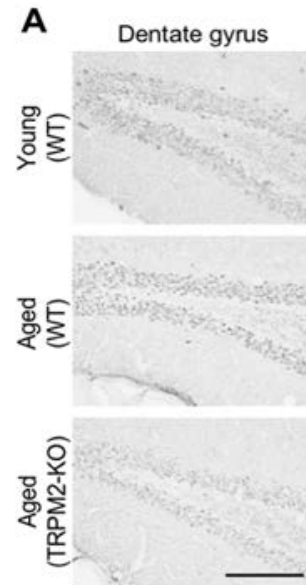

CA1

CA3

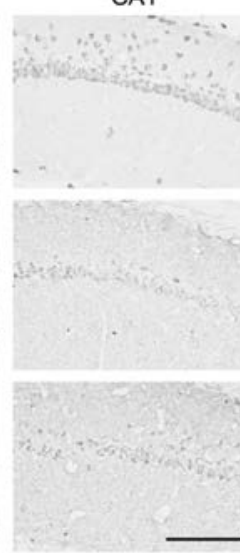

CA1 CA3

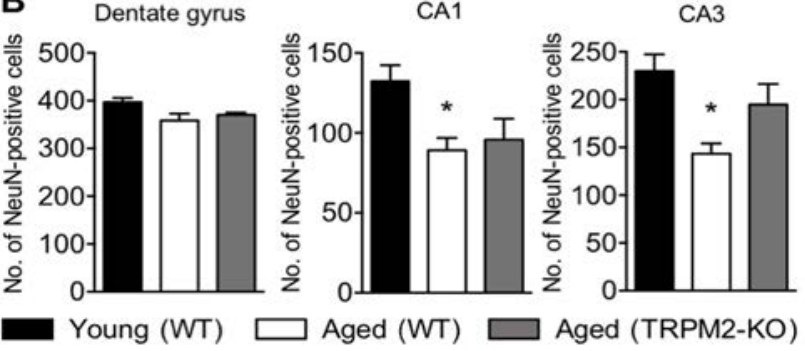

Fig. 4

A
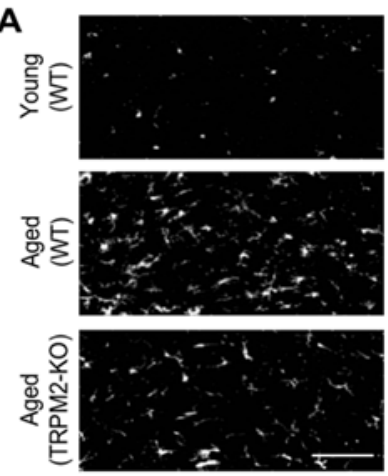

C
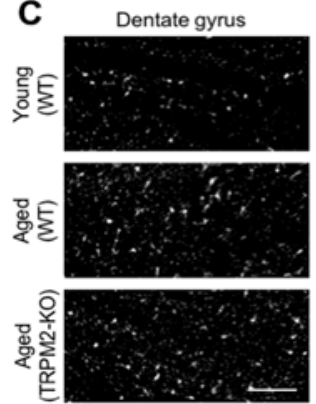

D Dentate gyrus
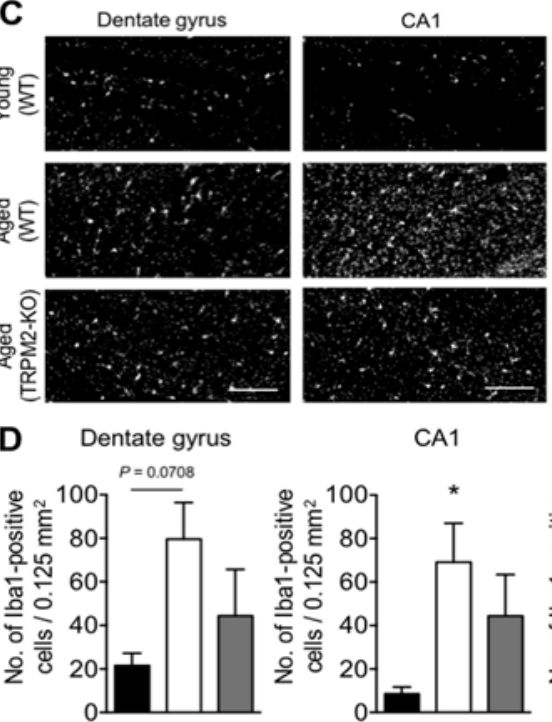

CA1

B Young (WT)

$\square$ Aged (WT)

$\square$ Aged (TRPM2-KO)

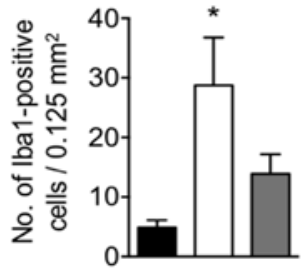

$\mathrm{CA} 3$
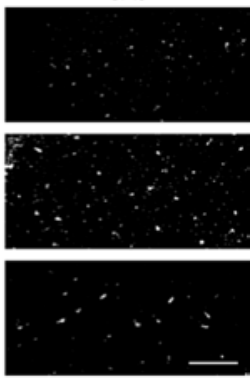

CA3

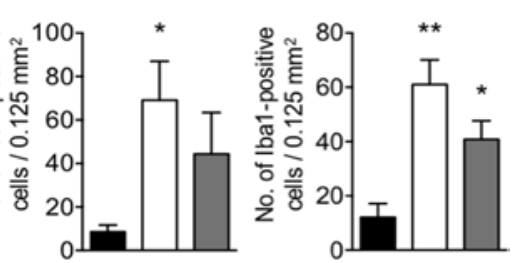


Fig. 5

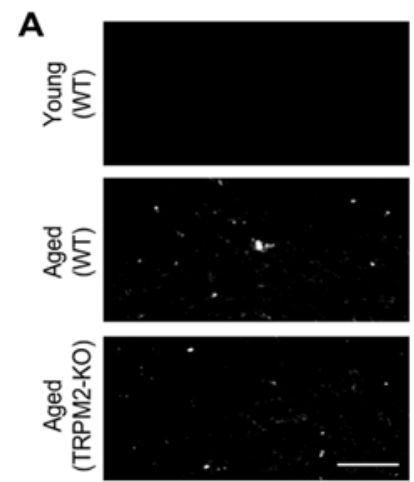

B Young (WT)

$\square$ Aged (WT)

$\square$ Aged (TRPM2-KO)
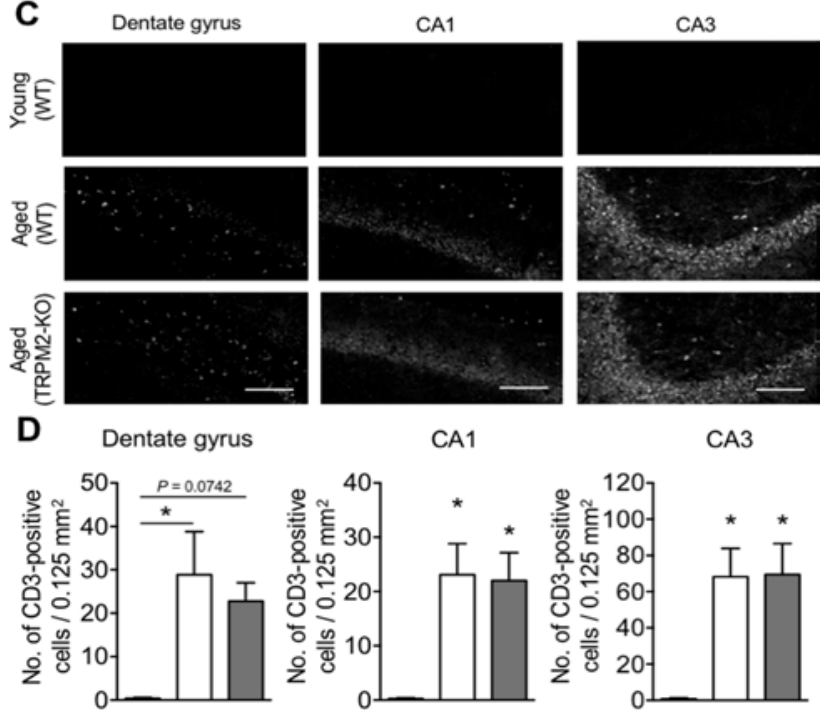

Fig. 6

Corpus Callosum
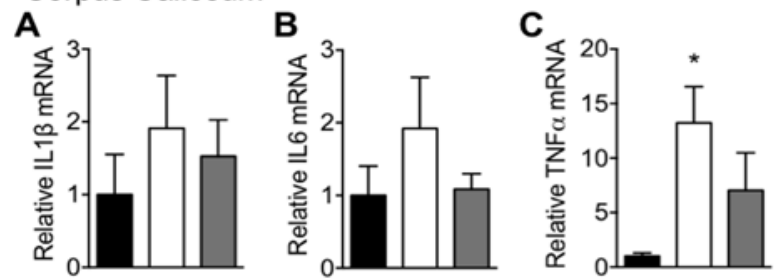

Hippocampus
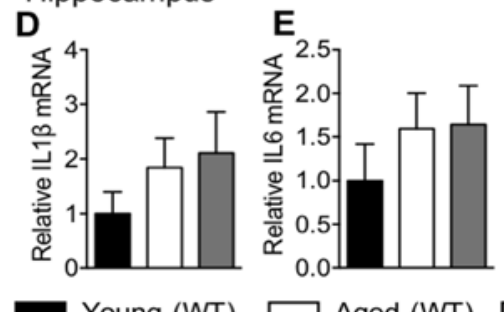

$\mathbf{F}$

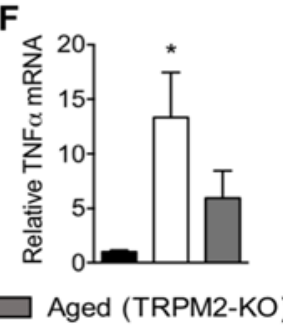




\section{Graphical Abstract}

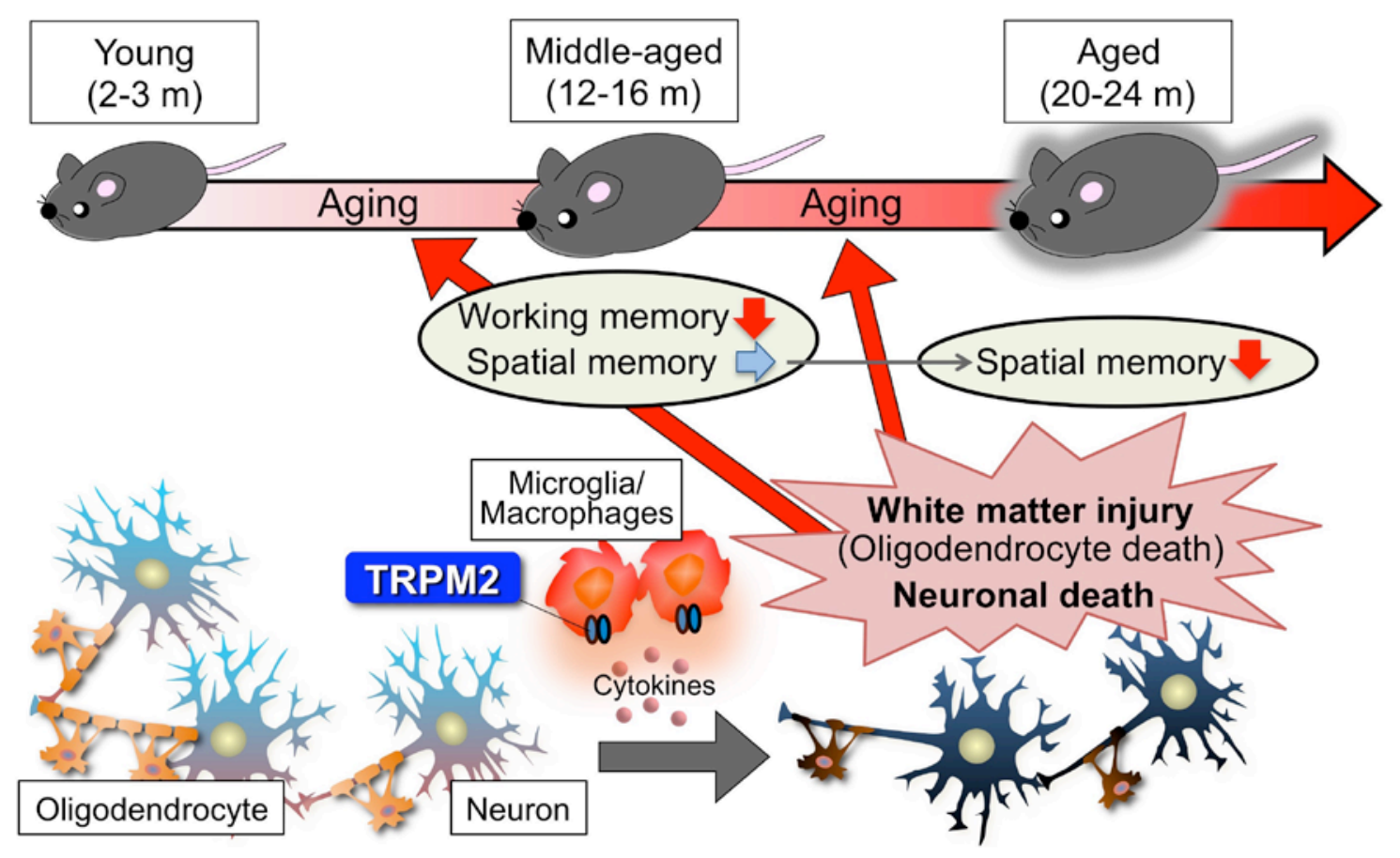

